\title{
Screening of potential biomarkers in the occurrence and development of type 1 diabetes mellitus based on transcriptome analysis
}

\author{
Jianhua Kang, Xingya Shen, Lishun Yang, Shaohua Feng, Delai Li, Haisheng Yuan \\ Department of Clinical Laboratory, Tianjin Beichen District Chinese Medicine Hospital, Tianjin, China
}

\begin{abstract}
Introduction: The aim of the study was to reveal the mechanisms for the pathogenesis and progression of type 1 diabetes mellitus (T1DM). Material and methods: Two mRNA expression profiles and two miRNA expression profiles were downloaded from the Gene Expression Omnibus (GEO) database. The differentially expressed genes (DEGs), differentially expressed miRNAs (DEMs), functional enrichment analyses, pathways, putative targets for DEMs and the miRNA-gene pairs, protein-protein pairs of DEGs, and PPI network were constructed. Results: Based on mRNA expression profiles, 37 and 110 DEGs were identified, and named as DEGs-short and DEGs-long, respectively. Based on miRNA expression profiles, 15 and six DEMs were identified, and named as DEMs-short and DEMs-long, respectively. DEGs-short were enriched in six GO terms and four pathways, and DEGs-long enriched in $40 \mathrm{GO}$ terms and 10 pathways. Seventeen miRNA-gene pairs for DEMs-short were screened out; hisa-miR-181a and hisa-miR-181c were involved in the most pairs. Twenty pairs for DEMs-long were obtained; $h s a-m i R-338-3 p$ was involved in all the pairs. KLRD1 was involved in more pairs in the network of DEGs-short. ACTA2 and USP9Y were involved in more pairs in the network of DEGs-long.

Conclusions: KLRD1, hisa-miR-181a, and hisa-miR-181c might be pathogenic biomarkers for T1DM, ACTA2, USP9Y, and hsa-miR-338-3p progressive biomarkers of T1DM. (Endokrynol Pol 2020; 71 (1): 58-65)
\end{abstract}

Key words: type 1 diabetes mellitus (T1DM); pathogenesis; progression; transcriptome analysis

\section{Introduction}

Diabetes mellitus (DM) is a group of metabolic disorders in which there are high blood sugar levels over a prolonged period. It is established that 415 million people had DM worldwide in 2015, and the number is predicted to reach more than 642 million by 2040 [1]. Moreover, from 2012 to 2015, approximately 1.5 to 5.0 million deaths each year resulted from DM [2]. DM is divided into three main types: Type 1 DM (T1DM), type $2 \mathrm{DM}$ (T2DM), and gestational diabetes. Type $1 \mathrm{DM}$ results from the pancreas's failure to produce enough insulin and makes up an estimated $5-10 \%$ of all diabetes cases [3]. The classical symptoms are frequent urination, increased thirst, increased hunger, and weight loss. At present, the cause of T1DM is still unknown, and genetic susceptibility, a diabetogenic trigger, and high exposure to an antigen are believed to be involved [4]. A meta-analysis involving 2238 T1DM participants showed that individuals had a higher risk for T1DM with the G allele of CTLA-4 +49A/G gene polymorphism [5]. Arroyo-Jousse et al. [6] found that T1DM patients showed a higher TNF $\alpha$ gene promoter methylation compared with control subjects [ $p=0.00008]$. A study of genome-wide gene expression analysis revealed that CD274 up-regulation in T1DM is correlated with the pathogenesis [7]. MicroRNA (miRNAs) are involved in various biological processes and become novel biomarkers in DM. A miRNA expression profile analysis showed that eight circulating miRNAs were dysregulated in T1DM patients (miR-21-5p, miR-146a-5p, miR-148a-3p, miR-181a-5p, miR-210-5p, miR-342-3p, miR-375, and miR-1275), which might be potential circulating biomarkers of this disease [8]. Moreover, a single-nucleotide polymorphism (rs2910164) in the miRNA-146a gene is significantly associated with diabetic nephropathy in T1DM patients [9].

Transcriptomics technologies are the techniques used to study an organism's transcriptome, the sum of all of its RNA transcripts. Among them, mRNA conveys genetic information from DNA to the ribosome, and miRNA functions in RNA silencing and post-transcriptional regulation of gene expression [10]. Transcriptomic analysis can study gene expression changes in different 
organisms, which contribute to the understanding of human disease [11]. In this study, the transcriptomic analyses were performed on new-onset and long-term T1DM patients in order to reveal the mechanisms for the pathogenesis and progression of this disease.

\section{Material and methods}

\section{Expression profiles}

The expression profiles of GSE55098 [12], GSE72492, GSE55099, and GSE97123 [13] were downloaded from the Gene Expression Omnibus (GEO) database (www.ncbi.nlm.nih.gov/geo/). The mRNA expression profile of GSE55098 contained 22 peripheral blood mononuclear cell (PBMC) samples from 12 newly diagnosed T1DM patients and 10 normal controls, and it was detected using the platform of [HG-U133_Plus_2] Affymetrix Human Genome U133 Plus 2.0 Array. The mRNA expression profile of GSE72492 included 17 pancreas tissue samples from T1DM patients and seven healthy humans, and these patients had been suffering from T1DM for at least five years. Agilent-028004 SurePrint G3 Human GE 8x60K Microarray was used to conduct the detection for GSE72492. Twelve PBMC samples from newly diagnosed T1DM patients and 10 PBMC samples from normal controls were contained in the miRNA expression profile of GSE55099, and they were sequenced with the platform of miRNA-1 Affymetrix Multispecies miRNA-1 Array. Twenty-four plasma-derived exosomes from 12 T1DM patients and 12 healthy patients were concluded in the miRNA expression profile of GSE97123, and these patients had suffered from T1DM for at least 25 years. The detection platform for GSE97123 was Counter Human miRNA Expression Assay.

\section{Data processing}

For GSE55098 and GSE55099, background correction, standardisation, and expression value calculation for the raw data were conducted with the affy V1.48.0 package (http://www.bioconductor. org/packages/3.2/bioc/html/affy.html). The hgu133plus2.db package V3.2.2 (http://www.bioconductor.org/packages/3.2/data/annotation/ $\mathrm{html} / \mathrm{hgu} 133$ plus2.db.html) was used to annotate, and the nonannotated probes were removed. For GSE72492, standardisation and logarithm calculation of expression values were performed with preprocessCore V1.32.0 (http://www.bioconductor.org/packages/3.2/bioc/html/preprocessCore.html). For GSE97123, the downloaded raw data had been normalised, and logarithm calculation was directly conducted.

\section{Differentially expressed analysis}

Based on the mRNA and miRNA profiles of GSE55098 and GSE55099, the differentially expressed genes (DEGs) and the differentially expressed miRNAs (DEMs) were separately identified in samples from newly diagnosed T1DM patients compared with those from normal controls with limma V3.32.2 (http://www.bioconductor.org/packages/3.5/bioc/html/limma.html), which were named as DEGs-short and DEMs-short, respectively. Furthermore, the DEGs and the DEMs were separately identified in samples from longstanding T1DM patients compared with those from healthy people in GSE72492 and GSE97123, and named as DEGs-long and DEMs-long, respectively. The threshold criteria was $\mid \log$ (fold change) $\mid>1$ and $\mathrm{p}<0.05$.

\section{Functional and pathway enrichment analyses of DEGs}

The functional enrichment analyses of the DEGs-short and DEGslong were performed via the Database for Annotation, Visualisation, and Integrated Discovery (DAVID) V6.8 (http://david.abcc.ncifcrf. gov/). The enriched pathway terms were screened out with the
Kyoto Encyclopaedia of Genes and Genomes (KEGG) PATHWAY (http://www.genome.jp/kegg), and Reactome (http://www.reactome.org). The threshold was $\mathrm{P}<0.05$.

\section{Targets prediction for DEMs}

Potential targets for DEMs-short and DEMs-long were predicted by $>5$ bioinformatics algorithms among the 10 algorithms in the miRWalk database: miRWalk V2.0 (www.ma.uni-heidelberg. de/apps/zmf/mirwalk/mirwalk), RNAhybrid V2.1 (www.ma.uniheidelberg.de/apps/zmf/mirwalk/rnahybrid), DIANAmT V4.0 (www.ma.uni-heidelberg.de/apps/zmf/mirwalk/diana-microt), miRanda -rel2010 (www.ma.uni-heidelberg.de/apps/zmf/mirwalk/ miranada), miRDB V4.0 (www.ma.uni-heidelberg.de/apps/zmf/ mirwalk/mirdb), PICTAR4 (www.ma.uni-heidelberg.de/apps/zmf/ mirwalk/pictar4), PICTAR5 (www.ma.uni-heidelberg.de/apps/zmf/ mirwalk/pictar5), PITA (www.ma.uni-heidelberg.de/apps/zmf/ mirwalk/pipa), RNA22 V2 (www.ma.uni-heidelberg.de/apps/zmf/ mirwalk/rna22), and Targetscan V6.2 (www.ma.uni-heidelberg.de/ apps/zmf/mirwalk/targetscan). Moreover, the negative regulated miRNA-gene pairs were selected out.

\section{The PPI network construction}

The Search Tool for the Retrieval of Interacting Genes/Proteins (STRING) is a biological database and web resource of known and predicted protein-protein interactions (PPI). The protein-protein pairs of DEMs-short and DEMs-long were identified via STRING v10.5 (https://string-db.org/) with more than 500 scores. Afterwards, the PPI networks for DEGs-short and DEGs-long were constructed and visualised by Cytoscape V3.5.1 software (http://www.cytoscape. org/download.php).

\section{Results}

\section{DEGs and DEMs}

After differentially expressed analysis, 37 (25 up- and 12 down-regulated) and 110 (58 up- and 52 down-regulated) DEGs were identified in sets of DEGs-short and DEGs-long, respectively; and 15 (two up- and 13 down-regulated) and six (one up- and five down-regulated) DEMs were identified in sets of DEMs-short and DEMs-long, respectively. Furthermore, the top 30 most significant DEGs of DEGs-short and DEGs-long are separately shown in Table 1 and Table 2, and all the DEMs of DEMs-short and DEMs-long are shown separately in Table 3 and Table 4 . Also, the overlaps of DEGs-short and DEGs-long were EIF1AY, LTF, and DDX3Y, and there was no overlap between DEMs-short and DEMs-long.

\section{The enriched gene ontology (GO) terms and pathways}

DEGs-short and DEGs-long were separately enriched in six and 40 GO terms, and all the gene ontology (GO) terms of DEGs-short and the top 10 most significant terms of DEGs-long are shown in Table 5 and Table 6, respectively. Moreover, DEGs-short were enriched in four pathway terms, namely "graft-versus-host disease", "antigen processing and presentation", "natural killer cell mediated cytotoxicity", and "signalling in immune system". DEGs-long were enriched in 10 pathways; namely, 
Table 1. The top 30 most significant differentially expressed genes (DEGs) in peripheral blood mononuclear cell samples from newly diagnosed patients with type 1 diabetes mellitus (T1DM) compared with those from normal controls (DEGs-short)

\begin{tabular}{lccccccc}
\hline Gene & LogFC & AveExpr & p value & Gene & LogFC & AveExpr & p value \\
\hline CD160 & -1.485740817 & 9.375468318 & $5.04 \mathrm{E}-06$ & CMTM2 & 1.43081945 & 8.187681273 & 0.006335067 \\
\hline KLRD1 & -1.127278189 & 8.941906288 & $1.10 \mathrm{E}-05$ & KCNJ2 & 1.077869083 & 6.547479591 & 0.006475015 \\
\hline CCL4 & -1.266147883 & 10.58044818 & $1.64 \mathrm{E}-05$ & CHI3L1 & 1.1924713 & 5.115614424 & 0.007671493 \\
\hline GNLY & -1.144524117 & 12.24886857 & $3.63 \mathrm{E}-05$ & CYP4F3 & 1.833172833 & 5.909274955 & 0.008561103 \\
\hline GZMH & -1.17887135 & 11.65018586 & $4.33 \mathrm{E}-05$ & S100P & 1.634646917 & 8.568421091 & 0.012775649 \\
\hline CLIC3 & -1.179784483 & 9.144989091 & $6.78 \mathrm{E}-05$ & KRT23 & 1.515719783 & 5.298236045 & 0.014109852 \\
\hline KLRF1 & -1.000714933 & 10.69416009 & 0.000117664 & CRISP3 & 1.10133205 & 4.734271773 & 0.02081107 \\
\hline FGFBP2 & -1.16267335 & 11.11717164 & 0.000195317 & SLPI & 1.387085033 & 6.380050636 & 0.022275293 \\
\hline KLRC3 & -1.267866217 & 8.382717909 & 0.000256813 & TNFAIP6 & 1.364051308 & 5.903216886 & 0.022326902 \\
\hline HLA-DOA1 & -1.78124685 & 5.714109242 & 0.001618603 & CEACAM8 & 1.822226467 & 7.058930909 & 0.026668873 \\
\hline DUSP2 & -1.289584217 & 8.945554227 & 0.001857457 & LTF & 1.407460333 & 9.775831136 & 0.029817392 \\
\hline CCR3 & 1.214328983 & 6.790913364 & 0.002497141 & RETN & 1.050559983 & 8.875287318 & 0.030854117 \\
\hline IFNG & -1.060417167 & 7.499563636 & 0.004650993 & MIMP & 1.185383167 & 7.671720636 & 0.030963045 \\
\hline FFAR2 & 1.626123483 & 5.858363545 & 0.004965728 & PGLYRP1 & 1.002344033 & 6.662580909 & 0.03233145 \\
\hline MS4A3 & 1.222284567 & 8.345452523 & 0.006033224 & RNASE3 & 1.3085789 & 7.113363909 & 0.034102172 \\
\hline
\end{tabular}

Table 2. The top 30 most significant differentially expressed genes (DEGs) in pancreas tissue samples from long-standing patients with type 1 diabetes mellitus (T1DM) compared with those from healthy people (DEGs-long)

\begin{tabular}{lccccccc}
\hline Gene & LogFC & AveExpr & p value & Gene & LogFC & AveExpr & p value \\
\hline INS & -7.956409294 & 11.80539147 & $1.61 \mathrm{E}-07$ & SYT7 & -1.030068536 & 7.626796818 & 0.000343623 \\
\hline IAPP & -4.973887634 & 8.748584024 & $6.19 \mathrm{E}-07$ & ANGPT2 & 1.148873747 & 7.086680079 & 0.000482281 \\
\hline SSC4D & -1.075597447 & 10.20799228 & $1.77 \mathrm{E}-06$ & EIF1AY & 1.08379585 & 7.046791771 & 0.000545989 \\
\hline CHST8 & -1.175932564 & 7.582315976 & $2.88 \mathrm{E}-06$ & HSPB2 & 1.104037749 & 8.503654391 & 0.000556139 \\
\hline INS-IGF2 & -3.232225473 & 6.533042141 & $3.07 \mathrm{E}-06$ & SLC25A34 & -1.728247111 & 10.11133012 & 0.000591585 \\
\hline PHYHIPL & 1.18534172 & 7.252759135 & $1.51 \mathrm{E}-05$ & CCDC3 & 1.05894566 & 9.655930312 & 0.000628911 \\
\hline GCGR & -1.70586376 & 6.827750629 & $2.11 \mathrm{E}-05$ & PTGIS & 1.424889976 & 8.260255438 & 0.000790294 \\
\hline SLC35D3 & -1.679842289 & 6.656338606 & $2.39 \mathrm{E}-05$ & DDX3Y & 1.992255074 & 6.248943021 & 0.000831372 \\
\hline G6PC2 & -2.116813487 & 6.112831353 & $3.10 \mathrm{E}-05$ & SLC30A8 & -1.494275889 & 9.141968897 & 0.000835193 \\
\hline SYT13 & -1.662898167 & 8.209265965 & $3.21 \mathrm{E}-05$ & RPS4Y1 & 5.34177196 & 8.2755228 & 0.000839019 \\
\hline EPN3 & -1.014739304 & 8.715785241 & $6.32 \mathrm{E}-05$ & UTY & 1.428451427 & 5.672497188 & 0.000859705 \\
\hline VEGFC & 1.186622956 & 8.308787518 & 0.00012934 & CCL21 & 2.10357751 & 8.799136812 & 0.000869965 \\
\hline CELF4 & -1.049638234 & 8.260741659 & 0.000138073 & FAM159B & -1.685486241 & 7.293984476 & 0.000950918 \\
\hline ADCYAP1 & -1.229689263 & 5.711479118 & 0.000153888 & UCHL1 & -1.205710231 & 9.847092909 & 0.000976663 \\
\hline PCSK1 & -1.979802762 & 8.473264497 & 0.000227194 & RPS4Y2 & 5.264040346 & 8.251606735 & 0.001024289 \\
\hline
\end{tabular}

"smooth muscle contraction", "vascular smooth muscle contraction", "regulation of insulin-like growth factor (IGF) transport and uptake by insulin-like growth factor binding proteins (IGFBPs)", "cGMP-PKG signalling pathway", "RHO GTPases activate PAKs", "platelet degranulation", "insulin processing", " chemical carcinogenesis", "antagonism of activin by follistatin", and "insulin secretion".

\section{The miRNA-gene pairs}

A total of 17 miRNA-gene pairs were screened out for DEMs-short, and they are shown in Table 7, including nine negatively regulated pairs. Moreover, hisa-miR-181a and hisa-miR-181c were involved in the most pairs. Twenty miRNA-gene pairs in total were screened out for DEMs-long (Tab. 8), including 11 
Table 3. All the differentially expressed miRNAs (DEMs) in peripheral blood mononuclear cell samples from newly diagnosed patients with type 1 diabetes mellitus (T1DM) compared with those from normal controls (DEMs-short)

\begin{tabular}{lccc}
\hline Gene & LogFC & AveExpr & p value \\
\hline hsa-miR-28-3p & -1.928257799 & 2.792230233 & $2.80 \mathrm{E}-06$ \\
\hline hsa-miR-146b-5p & -2.666591212 & 4.4375168 & $1.12 \mathrm{E}-05$ \\
\hline hsa-miR-181a-2 & -1.887221452 & 2.705472828 & $6.15 \mathrm{E}-05$ \\
\hline hsa-miR-28-5p & -1.383941091 & 3.923182481 & $6.17 \mathrm{E}-05$ \\
\hline hsa-miR-1225-3p & 1.023616193 & 1.73814695 & $9.27 \mathrm{E}-05$ \\
\hline hsa-miR-181c & -1.26274394 & 2.593748088 & 0.000439044 \\
\hline hsa-miR-1249 & 1.091444886 & 0.000543799 \\
\hline hsa-miR-199a-5p & -1.287916775 & 2.36209401 & 0.000747813 \\
\hline hsa-miR-125b & -1.075210791 & 1.850112207 & 0.000922042 \\
\hline hsa-miR-19b & -1.14946891 & 1.90891557 & 0.001454218 \\
\hline hsa-let-7f & -1.664920459 & 5.355734538 & 0.001554297 \\
\hline hsa-miR-487b & -1.334061912 & 5.356012127 & 0.012515649 \\
\hline hsa-miR-342-5p & -1.333040563 & 2.992923132 & 0.013564947 \\
\hline hsa-miR-30c & -1.050089726 & 5.825155044 & 0.01445844 \\
\hline hsa-miR-494 & -1.125921065 & 5.567514099 & 0.033330803 \\
\hline
\end{tabular}

Table 4. All the differentially expressed miRNAs (DEMs) in peripheral blood mononuclear cell samples from long-standing patients with type 1 diabetes mellitus (T1DM) compared with those from healthy people (DEMs-long)

\begin{tabular}{lccc}
\hline Gene & LogFC & AveExpr & p value \\
\hline hsa-miR-378e & -1.194166667 & 10.26958333 & $2.99 \mathrm{E}-06$ \\
\hline hsa-miR-338-3p & -1.083333333 & 6.218333333 & $1.10 \mathrm{E}-05$ \\
\hline hsa-miR-26a-5p & -1.019166667 & 4.987083333 & $2.58 \mathrm{E}-05$ \\
\hline hsa-miR-16-5p & -1.310833333 & 7.647083333 & 0.001228264 \\
\hline hsa-miR-144-3p & 1.811666667 & 1.316666667 & 0.025897521 \\
\hline hsa-miR-451a & -1.174166667 & 10.32875 & 0.031611088 \\
\hline
\end{tabular}

Table 5. All the enriched gene ontology (GO) terms of DEGs-short

\begin{tabular}{lccc}
\hline Category & Term & Gene count & p value \\
\hline BP & G0:0042742 defense response to bacterium & 6 & $2.13 \mathrm{E}-06$ \\
\hline BP & G0:0006935 chemotaxis & 5 & $2.40 \mathrm{E}-04$ \\
\hline CC & G0:0031226 intrinsic to plasma membrane & 7 & 0.012653662 \\
\hline MF & G0:0032393 $\sim$ MHC class I receptor activity & 2 & 0.03693037 \\
\hline BP & G0:0006026 aminoglycan catabolic process & 2 & 0.040243639 \\
\hline CC & G0:0005887 integral to plasma membrane & 6 & 0.042766631 \\
\hline
\end{tabular}

DEGs — differentially expressed genes; $\mathrm{BP}$ — biological process; CC — cellular component; MF — molecular function

negatively regulated pairs. Also, hsa-miR-338-3p was involved in all the above 20 miRNA-gene pairs.

\section{The PPI network}

After STRING screening, 19 and 89 protein-protein pairs of DEGs-short and DEGs-long were separately obtained, and the PPI networks of them are shown in Figure 1 and 2, respectively. The above pairs were a clustered different functional group in the networks, and KLRD1 (dark red) was involved in more pairs in a functional group of Figure 1, and ACTA2 and USP9Y (dark red) were involved in more pairs in different functional groups of Figure 2. 
Table 6. The top 10 most significantly enriched gene ontology (GO) terms of DEGs-long

\begin{tabular}{lccc}
\hline Category & Term & Gene count & p value \\
\hline CC & G0:0043292 contractile fibre & 9 & $3.40 \mathrm{E}-07$ \\
\hline CC & G0:0044444 cytoplasmic part & 46 & $3.35 \mathrm{E}-05$ \\
\hline BP & G0:0008217 regulation of blood pressure & 7 & $3.44 \mathrm{E}-05$ \\
\hline CC & G0:0030485 smooth muscle contractile fibre & 3 & $1.81 \mathrm{E}-04$ \\
\hline CC & G0:0005737 cytoplasm & 57 & $3.52 \mathrm{E}-04$ \\
\hline BP & G0:0031032 actomyosin structure organization & $6.66 \mathrm{E}-04$ \\
\hline CC & G0:0031410 cytoplasmic vesicle & 4 & $7.96 \mathrm{E}-04$ \\
\hline CC & G0:0030016 myofibril & 5 & 0.003350748 \\
\hline BP & G0:0030334 regulation of cell migration & 6 & 0.003896976 \\
\hline BP & G0:0048738 cardiac muscle tissue development & 4 & 0.005230827 \\
\hline
\end{tabular}

DEGs — differentially expressed genes; BP — biological process; CC — cellular component

Table 7. The miRNA-gene pairs of DEMs-short

\begin{tabular}{lccc}
\hline MicroRNA & Gene & MicroRNA_logFC & Gene_logFC \\
\hline hsa-miR-181c & TNFAIP6 & -1.26274394 & 1.364051308 \\
\hline hsa-miR-181c & DDX3Y & -1.26274394 & 1.249616883 \\
\hline hsa-miR-181c & KCNJ2 & -1.26274394 & 1.077869083 \\
\hline hsa-miR-181a & TNFAIP6 & -1.887221452 & 1.364051308 \\
\hline hsa-miR-181a & DDX3Y & -1.887221452 & 1.249616883 \\
\hline hsa-miR-181a & KCNJ2 & -1.887221452 & 1.077869083 \\
\hline hsa-miR-146b-5p & EIF1AY & 1.9180783 \\
\hline hsa-miR-146b-5p & CEACAM8 & -2.666591212 & 1.822226467 \\
\hline hsa-miR-125b & MS4A3 & -2.666591212 & 1.2222845567 \\
\hline hsa-miR-181c & KLRF1 & -1.075210791 & -1.000714933 \\
\hline hsa-miR-181a & KLRF1 & -1.26274394 & -1.000714933 \\
\hline hsa-miR-181c & IFNG & -1.887221452 & -1.060417167 \\
\hline hsa-miR-181a & IFNG & -1.26274394 & -1.060417167 \\
\hline hsa-miR-125b & IFNG & -1.887221452 & -1.060417167 \\
\hline hsa-miR-146b-5p & FGFBP2 & -1.075210791 & -1.16267335 \\
\hline hsa-miR-125b & CCL4 & -2.666591212 & -1.266147883 \\
\hline hsa-let-7f & DUSP2 & -1.075210791 & -1.289584217 \\
\hline
\end{tabular}

DEMs - differentially expressed miRNAs

\section{Discussion}

Many genes and miRNAs have been indicated to be involved in the occurrence and development of T1DM. In this study, we parallelly identified and analysed the differential expressions for newly diagnosed and long-standing T1DM patients. Afterwards, hisa-miR-181a and hisa-miR-181c were found to be involved in the most miRNA-gene pairs of DEMs-short (Tab. 7), and the node of KLRD1 was involved in the most pairs in the PPI network of DEGs-short (Fig. 1). A meta-analysis showed that the hsa-miR-181 family are involved in the inhibition of IL-2 expression, and hisa-miR-181a and hisa-miR-181c contribute to T cell tolerance, which is very important in the pathogenesis and treatment of T1DM [14, 15]. Also, the meta-analysis also proved that hsa-miR-181c was differentially expressed in the three types of diabetes (T1DM, T2DM, and gestational diabetes). Another study found that hsa-miR-181c was down-regulated in a diabetic-like environment and up-regulated after the addition of calcitriol [16]. Endothelial dysfunction played an important role in the occurrence and development of $\mathrm{DM}$, and hisa-miR-181c could attenuate nitration stress 
Table 8. The miRNA-gene pairs of DEMs-long

\begin{tabular}{lccc}
\hline MicroRNA & Gene & MicroRNA_logFC & Gene_logFC \\
\hline hsa-miR-338-3p & CCL21 & -1.083333333 & 2.10357751 \\
\hline hsa-miR-338-3p & CXCL9 & -1.083333333 & 1.77456068 \\
\hline hsa-miR-338-3p & ALDOB & -1.083333333 & 1.580524957 \\
\hline hsa-miR-338-3p & UTY & -1.083333333 & 1.428451427 \\
\hline hsa-miR-338-3p & PTGIS & -1.083333333 & 1.424889976 \\
\hline hsa-miR-338-3p & PRRX1 & 1.242794076 \\
\hline hsa-miR-338-3p & TMOD1 & -1.083333333 & 1.196113314 \\
\hline hsa-miR-338-3p & HOXA3 & -1.083333333 & 1.121206093 \\
\hline hsa-miR-338-3p & TFPI & -1.083333333 & 1.100948365 \\
\hline hsa-miR-338-3p & EIF1AY & -1.083333333 & 1.08379585 \\
\hline hsa-miR-338-3p & COL12A1 & -1.083333333 & 1.040322198 \\
\hline hsa-miR-338-3p & SYT7 & -1.083333333 & -1.030068536 \\
\hline hsa-miR-338-3p & RGS16 & -1.083333333 & -1.031304186 \\
\hline hsa-miR-338-3p & AOP2 & -1.083333333 & -1.034998067 \\
\hline hsa-miR-338-3p & PPP1R1A & -1.083333333 & -1.113167906 \\
\hline hsa-miR-338-3p & UNC5A & -1.083333333 & -1.173571 \\
\hline hsa-miR-338-3p & SLC30A8 & -1.083333333 & -1.494275889 \\
\hline hsa-miR-338-3p & SYT13 & -1.083333333 & -1.662898167 \\
\hline hsa-miR-338-3p & SLC25A34 & -1.728247111 \\
\hline hsa-miR-338-3p & PCSK1 & -1.083333333 & -1.979802762 \\
\hline
\end{tabular}

DEMs - differentially expressed miRNAs
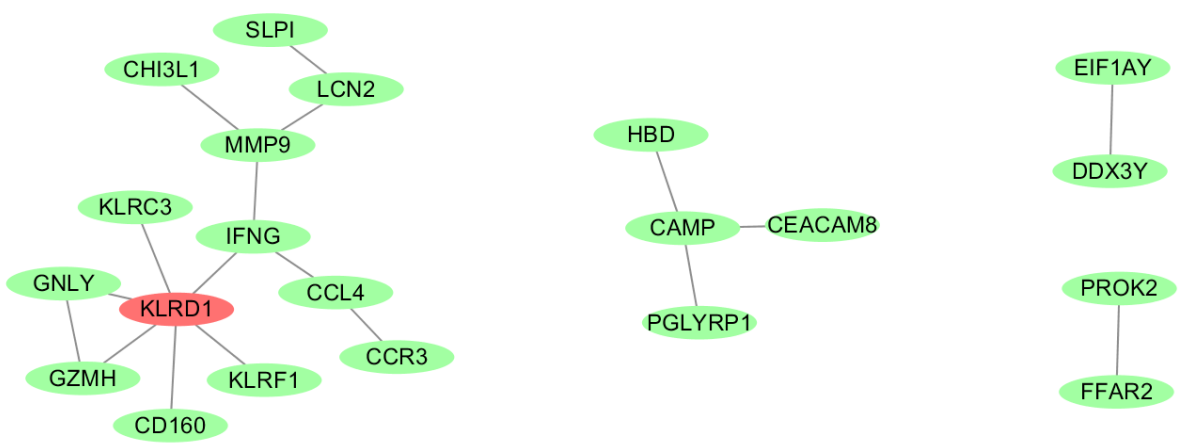

Figure 1. The protein-protein interaction (PPI) network of DEGs-short

through regulating FoxO1 expression and affecting endothelial cell function [17]. It might be one of the mechanisms of hisa-miR-181c in the occurrence and development of DM. Killer cell lectin-like receptor subfamily D, member 1 (KLRD1), encoded by KLRD1 gene, is an antigen preferentially expressed on NK cells, and also known as cluster of differentiation 94 (CD94). Nakata et al. [18] reported that the expression of KLRD1 was reduced in NK-enriched cells in fulminant T1DM. Goodier et al. [19] reported that there was a significant reduction in the proportion of CD94 $(+)$ cells responding to lipopolysaccharide in T1DM compared to the non-diabetic twin $(\mathrm{p}=0.025)$, which might be associated with the cause of T1DM. Therefore, we suspected that KLRD1, hisa-miR-181a, and hisa-miR-181c were novel biomarkers in the pathogenesis of T1DM. Also, this article identified some targets for hisa-miR-181a and hisa-miR-181c, such as KCNJ2, DDX3Y, KLRF1, IFNG, etc. (Tab. 7).

Furthermore, our results showed that hsa-miR-338-3p was involved in all the miRNA-gene pairs of DEMs-long (Tab. 8). The PPI network of DEGs-long was clustered different functional groups, and ACTA2 and USP9Y were involved in more pairs in different 

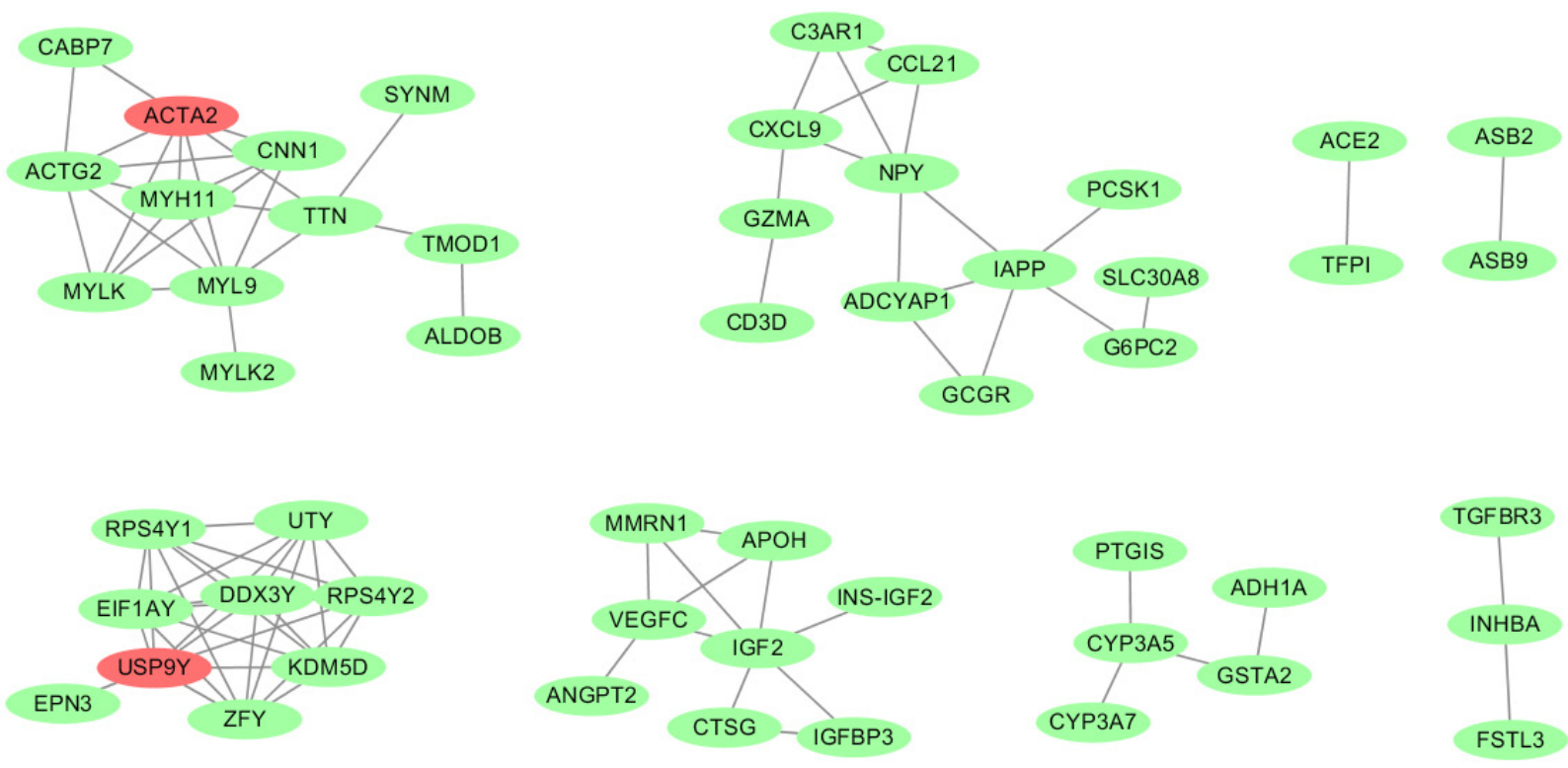

Figure 2. The protein-protein interaction (PPI) network of DEGs-long

functional groups (Fig. 2). Jacovetti et al. [20] found in rodents that $\beta$ cell mass expansion during pregnancy and obesity is associated with the expression change of hsa-miR-338-3p; they also revealed a major role for hsa-miR-338-3p in compensatory $\beta$ cell mass expansion occurring under different insulin resistance states. Subsequently, Nesca et al. [21] reported that the expression hsa-miR-338-3p displayed changes occurring before the onset of diabetes, which were positive effects on $\beta$ cell activities and mass; in contrast, modification in the level of hsa-miR-338-3p primarily occurred in diabetic mice and resulted in increased $\beta$ cell apoptosis. These results indicate that the expression change of hsa-miR-338-3p participates in the progression of diabetes. Alpha-actin-2 ( $\alpha$-SMA) is a protein encoded by the ACTA2 gene, which is commonly used as a marker of myofibroblast formation [22]. ACTA2 is the human aortic smooth muscle actin gene and is involved in cell motility, structure, and integrity [23]. Moreover, DEGs-long was enriched in GO terms of "contractile fibre", "smooth muscle contractile fibre", "actomyosin structure organisation", "myofibril", and "cardiac muscle tissue development" (Tab. 6), and ACTA2 played very important roles in the above GO terms. Although few reports revealed the relationship between ACTA2 and T1DM, our results suggest that ACTA2 is associated with the progression of this disease. USP9Y gene encodes the enzyme of ubiquitin specific peptidase 9, Y-linked (USP9Y), which locates on the $\mathrm{Y}$ chromosome. Mutations in this gene are associated with Sertoli cell-only syndrome (SCO) and male infertility. The gene fusion TTTY15-USP9Y score was statistically significantly higher in prostate cancer men with positive biopsy outcome than in men with negative biopsy outcome ( $p<0.001)$, and thus TTTY15-USP9Y could be used to predict biopsy outcome [24]. USP9Y presents only in black people of African origin and attributes a favourable lipoprotein pattern, which is very important in the development of diabetes [25]. Previously, there was no direct evidence that USP9Y is associated with TIDM. Here, our article found that USP9Y occupied a critical position in the PPI network of DEGs-long, which suggested that USP9Y might play a role in the development of T1DM.

\section{Conclusion}

In conclusion, our study suggested that KLRD1, hisa-miR-181a, and hisa-miR-181c were involved in the onset of T1DM, and that ACTA2, USP9Y, and hsa-miR-338-3p played some important roles in its development. They are potential biomarkers in the pathogenesis or progression of T1DM, which provides further insights for T1DM.

\section{Funding}

This work was supported by the program of Beichen District Science and Technology Commission, Tianjin (grant 2016-SHGY-13).

\section{Availability of data and material \\ Not applicable.}

\section{Acknowledgements}

We would like to thank all the members of our research group for their enthusiastic participation in this study. 


\section{Competing interests}

The authors declare that they have no competing interests.

\section{Consent for publication \\ Not applicable.}

\section{Ethics approval and consent to participate Not applicable.}

\section{References}

1. Iwahashi H, Imagawa A. Type 2 diabetes mellitus prevention strategy: Target and goal in Japanese men. J Diabetes Investig. 2016; 7(3): 283-285, doi: 10.1111/jdi.12481, indexed in Pubmed: 27330711.

2. Treating the Inflammatory Core of Metabolic Syndromes. EBioMedicine. 2015; 2(9): 1003-1004, doi: 10.1016/j.ebiom.2015.09.011, indexed in Pubmed: 26501087.

3. Daneman D. Type 1 diabetes. The Lancet. 2006; 367(9513): 847-858, doi: 10.1016/s0140-6736(06)68341-4.

4. Knip M, Veijola R, Virtanen SM, et al. Environmental triggers and determinants of type 1 diabetes. Diabetes. 2005; 54 Suppl 2: S125-S136, doi: 10.2337/diabetes.54.suppl 2.s125, indexed in Pubmed: 16306330.

5. Li Yy, Gong Ge, Geng Hy, et al. CTLA- $4+49 \mathrm{~A} / \mathrm{G}$ gene polymorphism and type 1 diabetes mellitus in the Chinese population: a meta-analysis of 2238 subjects. International Journal of Diabetes in Developing Countries. 2015; 36(1): 45-51, doi: 10.1007/s13410-015-0414-0.

6. Arroyo-Jousse V, Garcia-Diaz DF, Codner E, et al. Epigenetics in type 1 diabetes: TNFa gene promoter methylation status in Chilean patients with type 1 diabetes mellitus. Br J Nutr. 2016; 116(11): 1861-1868, doi: 10.1017/S0007114516003846, indexed in Pubmed: 27890035

7. Fang C, Huang Y, Pei Y, et al. Genome-wide gene expression profiling reveals that CD274 is up-regulated new-onset type 1 diabetes mellitus. Acta Diabetol. 2017; 54(8): 757-767, doi: 10.1007/s00592-017-1005-y, indexed in Pubmed: 28577136.

8. Assmann TS, Recamonde-Mendoza M, De Souza BM, et al. MicroRNA expression profiles and type 1 diabetes mellitus: systematic review and bioinformatic analysis. Endocr Connect. 2017; 6(8): 773-790 doi: 10.1530/EC-17-0248, indexed in Pubmed: 28986402.

9. Kaidonis G, Gillies MC, Abhary S, et al. A single-nucleotide polymorphism in the MicroRNA-146a gene is associated with diabetic ne phropathy and sight-threatening diabetic retinopathy in Caucasian patients. Acta Diabetol. 2016; 53(4): 643-650, doi: 10.1007/s00592-016-0850-4 indexed in Pubmed: 26997512.

10. Ambros V. The functions of animal microRNAs. Nature. 2004; 431(7006): 350-355, doi: 10.1038/nature02871, indexed in Pubmed: 15372042.

11. Lowe R, Shirley N, Bleackley M, et al. Transcriptomics technologie. PLoS Comput Biol. 2017; 13(5): e1005457, doi: 10.1371/journal.pcbi.1005457, indexed in Pubmed: 28545146.
12. Yang $M$, Ye L, Wang B, et al. Decreased miR-146 expression in peripheral blood mononuclear cells is correlated with ongoing islet autoimmunity in type 1 diabetes patients 1miR-146. J Diabetes. 2015; 7(2): 158-165, doi: 10.1111/1753-0407.12163, indexed in Pubmed: 24796653.

13. Garcia-Contreras M, Shah SH, Tamayo A, et al. Plasma-derived exosome characterization reveals a distinct microRNA signature in long duration Type 1 diabetes. Sci Rep. 2017; 7(1): 5998, doi: 10.1038/s41598-017-05787-y, indexed in Pubmed: 28729721.

14. Collares, C, Evangelista A, Xavier D. Meta-analysis of differentially expressed microRNAs in type 1 , type 2 and gestational diabetes mellitus. Endocrine Abstracts. 2012; 29(OC17.6).

15. Saudek F, Havrdova T, Boucek P, et al. Polyclonal anti-T-cell therapy for type 1 diabetes mellitus of recent onset. Rev Diabet Stud. 2004; 1(2) 80-88, doi: 10.1900/RDS.2004.1.80, indexed in Pubmed: 17491669.

16. Zitman-Gal T, Green J, Pasmanik-Chor M, et al. Vitamin D manipulates miR-181c, miR-20b and miR-15a in human umbilical vein endothelial cells exposed to a diabetic-like environment. Cardiovasc Diabetol. 2014; 13: 8, doi: 10.1186/1475-2840-13-8, indexed in Pubmed: 24397367

17. Yang G, Wu Y, Ye S. MiR-181c restrains nitration stress of endothelia cells in diabetic $\mathrm{db} / \mathrm{db}$ mice through inhibiting the expression of FoxO1. Biochem Biophys Res Commun. 2017; 486(1): 29-35, doi: 10.1016/j. bbrc.2017.02.083, indexed in Pubmed: 28223216.

18. Nakata S, Imagawa A, Miyata Y, et al. Low gene expression levels of activating receptors of natural killer cells (NKG2E and CD94) in patients with fulminant type 1 diabetes. Immunol Lett. 2013; 156(1-2): 149-155 doi: 10.1016/j.imlet.2013.10.004, indexed in Pubmed: 24177169.

19. Goodier MR, Nawroly N, Beyan $\mathrm{H}$, et al. Identical twins discordant for type 1 diabetes show a different pattern of in vitro CD56+ cell activation. Diabetes Metab Res Rev. 2006; 22(5): 367-375, doi: 10.1002/dmrr.627, indexed in Pubmed: 16572491.

20. Jacovetti C, Abderrahmani A, Parnaud G, et al. MicroRNAs contribute to compensatory $\beta$ cell expansion during pregnancy and obesity. J Clin Invest. 2012; 122(10): 3541-3551, doi: 10.1172/JCI64151, indexed in Pubmed: 22996663.

21. Nesca V, Guay C, Jacovetti C, et al. Identification of particular groups of microRNAs that positively or negatively impact on beta cell function in obese models of type 2 diabetes. Diabetologia. 2013; 56(10): 2203-2212, doi: 10.1007/s00125-013-2993-y, indexed in Pubmed: 23842730.

22. Nagamoto T, Eguchi G, Beebe DC. Alpha-smooth muscle actin expression in cultured lens epithelial cells. Invest Ophthalmol Vis Sci. 2000; 41(5): 1122-1129, indexed in Pubmed: 10752950.

23. Wu Z, Wang S, Jiang F, et al. Mass spectrometric detection combined with bioinformatic analysis identified possible protein markers and key pathways associated with bladder cancer. Gene. 2017; 626: 407-413, doi: 10.1016/j.gene.2017.05.054, indexed in Pubmed: 28552713.

24. Zhu Y, Ren S, Jing T, et al. Clinical utility of a novel urine-based gene fusion TTTY15-USP9Y in predicting prostate biopsy outcome. Uro Oncol. 2015; 33(9): 384.e9-384.20, doi: 10.1016/j.urolonc.2015.01.019, indexed in Pubmed: 26008593.

25. Russo P, Siani A, Miller MA, et al. Genetic variants of Y chromosome are associated with a protective lipid profile in black men Arterioscler Thromb Vasc Biol. 2008; 28(8): 1569-1574, doi: 10.1161/ATVBAHA.108.168641, indexed in Pubmed: 18511697. 